\title{
GENERATING GROUPS OF NILPOTENT VARIETIES
}

BY L. G. KOVÁCS, M. F. NEWMAN AND P. F. PENTONY

Communicated by Walter Feit, March 21, 1968

Problem 14 of Hanna Neumann's book [3] asks for a proof of a conjecture which is contradicted by the following

THEOREM. If $c$ is an integer greater than 2 , then the variety $\mathfrak{N}_{c}$ of all nilpotent groups of class at most $c$ is generated by its free group $F_{c-1}\left(\mathfrak{N}_{c}\right)$ of rank $c-1$ but not by its free group $F_{c-2}\left(\mathfrak{N}_{c}\right)$ of rank $c-2$.

In the terms of [3], this means that for $c>2$ one has $d(c)=c-1$ rather than $d(c)=[c / 2]+1$ as suggested in Problem 14; correspondingly, $[3,35.35]$ is false for $c=5$ and 6 . (Professor Neumann has confirmed that her proofs were faulty.)

The theorem was suggested by Graham Higman's approach to nilpotent varieties of class $c$ and prime exponent greater than $c$, via the representation theory of the general linear groups [1]. In particular, he remarked that each critical group in such a variety can be generated by $c-1$ elements (if $c>2$ ). Since $F_{c}\left(\mathfrak{N}_{c}\right)$ generates $\mathfrak{N}_{c}$ (cf. $[3,35.12]$ ) and is residually of prime exponent (cf. Higman [2]), it follows easily that $F_{c-1}\left(\mathfrak{R}_{c}\right)$ generates $\mathfrak{R}_{c}$. It is not difficult to use Higman's method for confirming the second half of the theorem as well.

In this note we outline a proof which avoids the conceptual complexity of Higman's approach; the price of this is paid for in length. Unless otherwise specified, our notation and terminology follow Hanna Neumann's book [3].

To prove the first half of the theorem, it is sufficient to find a set of homomorphisms from $F_{c}\left(\mathfrak{N}_{c}\right)$ to $F_{c-1}\left(\mathfrak{N}_{c}\right)$ whose kernels intersect trivially. Hanna Neumann did just this in the proof of $[3,35.35]$ for $c=4$, and the same idea works generally: if $\left\{a_{1}, \cdots, a_{c}\right\}$ is a free generating set for $F_{c}\left(\mathfrak{R}_{c}\right)$ and $\left\{b_{1}, \ldots, b_{c-1}\right\}$ is one for $F_{c-1}\left(\mathfrak{n}_{c}\right)$, then the $2 c-1$ homomorphisms $\delta_{1}, \cdots, \delta_{c}, \theta_{1}, \cdots, \theta_{c-1}$ defined by

$$
\begin{aligned}
& a_{j} \delta_{i}=b_{j} \quad \text { if } j<i \text {, and } \quad a_{j} \theta_{i}=b_{j} \quad \text { if } j \leqq i, \\
& a_{j} \delta_{i}=1 \quad \text { if } j=i, \quad a_{j} \theta_{i}=b_{j-1} \text { if } j>i \\
& a_{j} \delta_{i}=b_{j-1} \text { if } j>i
\end{aligned}
$$

will do. The verification of this makes use of the unique representation of the elements of $F_{c}\left(\mathfrak{N}_{c}\right)$ in terms of basic commutators in $\left\{a_{1}, \cdots, a_{0}\right\}$ as defined by Martin Ward [4]. The case of odd $c$ is 
comparatively straightforward, that of even $c$ requires lengthy and careful discussion.

For the second half, we show that the word $w_{c}$ defined below is a law in $F_{c-2}\left(\mathfrak{R}_{c}\right)$, and then we exhibit a nilpotent group $G_{c}$ of class $c$ (and soluble length 3 ) in which $w_{c}$ is not a law. The choice of this word was suggested by Higman's approach. Let $S$ denote the group of all permutations of $\{1, \cdots, c-1\}$, and let $\epsilon(\sigma)$ be 1 or -1 according as $\sigma$ is an even or odd permutation; then

$$
w_{c}=\prod_{\sigma \in S}\left[x_{c}, x_{(c-1) \sigma}, \cdots, x_{1 \sigma}\right]^{e(\sigma)} .
$$

If $F=F_{c-2}\left(\mathfrak{N}_{c}\right)$ is freely generated by $\left\{a_{1}, \cdots, a_{c-2}\right\}$, then every element of $F$ can be written in the form

$$
d \prod_{j=1}^{c-2} a_{j}^{\alpha(j)}
$$

where $d$ belongs to the commutator subgroup $F^{\prime}$ of $F$. Substituting $d_{i} \prod_{j=1}^{c-2} a_{j}^{\alpha(i, j)}$ for $x_{i}$ in $w_{c}$, one finds that every value of $w_{c}$ in $F$ is of the form

$$
\prod_{\phi}\left[a_{c \phi}, a_{(0-1) \phi}, \cdots, a_{1 \phi}\right]^{\beta(\phi)}
$$

where $\phi$ runs through all maps from $\{1, \cdots, c\}$ to $\{1, \cdots, c-2\}$ and

$$
\beta(\phi)=\alpha(c, c \phi) \sum_{\sigma \in S} \epsilon(\sigma) \prod_{i=1}^{0-1} \alpha(i \sigma, i \phi) .
$$

Now two of $1 \phi, \cdots,(c-1) \phi$ must be equal: say, $r \phi=s \phi$. Let $T$ be a transversal of the subgroup of $S$ generated by the transposition ( $r s$ ) such that every element of $S$ not in $T$ can be written uniquely as $(r s) \tau$ with $\tau \in T$. Then

$$
\begin{aligned}
\beta(\phi) & =\alpha(c, c \phi) \sum_{\tau \in T} \epsilon(\tau)\left\{\prod_{i=1}^{c-1} \alpha(i \tau, i \phi)-\prod_{i=1}^{\infty-1} \alpha(i(r s) \tau, i \phi)\right\} \\
& =0 \quad \text { because } r \phi=s \phi .
\end{aligned}
$$

Thus $w_{c}$ is a law in $F$.

The choice of $G_{c}$ was influenced by the observation that $w_{c}$ can be rewritten as follows. Put $n=\left[\frac{1}{2}(c+1)\right]$ so that $c=2 n$ or $2 n-1$; note that $n>1$ as $c>2$. For each $j$ in $\{1, \cdots, n\}$, let $K_{j}$ be the subgroup of $S$ generated by the transpositions $(2 i-12 i)$ with $i \in\{j, \cdots, n-1\}$ : thus $K_{n}$ is the identity subgroup of $S$. Let $T$ denote an arbitrary transversal of $K_{1}$ in $S$ so that $S=K_{1} T$. Then $w_{c}=w_{c}^{T} d_{c}^{T}$ where 


$$
\begin{aligned}
w_{2 n}^{T} & =\prod_{\tau \in T}\left[\left[x_{2 n}, x_{(2 n-1) \tau}\right],\left[x_{(2 n-2) \tau}, x_{(2 n-3) \tau}\right], \cdots,\left[x_{2 \tau}, x_{1 \tau}\right]\right]^{e(\tau)}, \\
w_{2 n-1}^{T} & =\prod_{\tau \in T}\left[x_{2 n-1},\left[x_{(2 n-2) r}, x_{(2 n-3) \tau}\right], \cdots,\left[x_{2 r}, x_{1 \tau}\right]\right] \in(\tau)
\end{aligned}
$$

and $d_{c}^{T}$ is a law in $\mathfrak{N}_{c}$. This is a consequence of the fact that

$\left[y,\left[x_{2 n-2}, x_{2 n-3}\right], \cdots,\left[x_{2}, x_{1}\right]\right]$

$=d_{j} \prod_{\sigma \in K_{j}}\left[y, x_{(2 n-2) \sigma}, \cdots, x_{(2 j-1) \sigma},\left[x_{(2 j-2) \sigma}, x_{(2 j-3) \sigma}\right], \cdots,\left[x_{2 \sigma}, x_{1 \sigma}\right]\right] \cdot(\sigma)$

where $d_{j}$ is a law in $\mathfrak{N}_{c}:$ a tautology if $j=n$ and $d_{n}=1$, and proved in general by reverse induction on $j$.

The group $G_{c}$ is constructed as follows. Let $W_{n}=F_{2 n}\left(\mathfrak{R}_{n} \cap \mathfrak{2}^{2}\right)$ be freely generated by $C=\left\{u_{2}, u_{4}, \cdots, u_{2 n}, v_{1}, \cdots, v_{n}\right\}$. For each $i$ in $\{1, \cdots, n\}$, there is an automorphism $\gamma_{i}$ of $W_{n}$ which maps $u_{2 i}$ to $u_{2 i} v_{i}$ and fixes all other elements of $C$. It is easy to check that $\gamma_{1}, \cdots, \gamma_{n}$ generate a free abelian subgroup of rank $n$ in the automorphism group of $W_{n}$. The group $H_{n}$ is the splitting extension of $W_{n}$ by a free abelian group of rank $n$ freely generated by $u_{1}, u_{3}, \cdots, u_{2 n-1}$ where the homomorphism specifying the extension maps $u_{2 i-1}$ to $\gamma_{i}$ for all $i$. We take $G_{c}$ to be the largest nilpotent-of-class- $c$ factor group of the subgroup of $H_{n}$ generated by $\left\{u_{1}, \cdots, u_{c}\right\}$.

We now sketch the verification that this $G_{c}$ has the required properties. If $z_{1}, \cdots, z_{r} \in C$, then $\left[z_{1}, \cdots, z_{r}, u_{2 i-1}\right]$ is a (possibly empty) product of left-normed commutators of weight at least $r$ with entries from $C$ in which all the commutators of weight $r$ have fewer entries $u_{2 i}$ than $\left[z_{1}, \cdots, z_{r}\right]$. From this, a routine argument shows that every left-normed commutator of weight $2 n$ with entries from $\left\{u_{1}, \cdots, u_{2 n}\right\}$ lies in the $n$th term of the lower central series of the subgroup of $W_{n}$ generated by $\left\{v_{1}, \cdots, v_{n}\right\}$. It follows that $H_{n}$ has class $2 n$.

Suppose that $c=2 n$, so that $G_{c}=H_{n}$, and evaluate $w_{2 n}$ when $u_{i}$ is substituted for $x_{i}$. Since each $d_{2 n}^{T}$ is a law in $H_{n}$, we get the same value from substituting in any $w_{2 n}^{T}$, and we exploit our freedom in choosing $T$. Let $R$ be the group of all permutations on $\{1, \cdots, n-1\}$; define a monomorphism $\rho \rightarrow \rho^{*}$ from $R$ to $S$ by $(2 k-1) \rho^{*}=2(k \rho)-1,(2 k) \rho^{*}$ $=2(k \rho)$ if $k<n$, and $(2 n-1) \rho^{*}=2 n-1$, and denote its image by $R^{*}$. As $R^{*}$ avoids $K_{1}$, it can be extended to a transversal $T$. Observe that $\left[u_{i}, u_{j}\right]$ lies in the commutator subgroup of $W_{n}$ unless $|i-j|=1$ and $\max (i, j)$ is even; hence 


$$
\left[\left[u_{2 n}, u_{(2 n-1) \tau}\right],\left[u_{(2 n-2) \tau}, u_{(2 n-3) \tau}\right], \cdots,\left[u_{2 r}, u_{1 \tau}\right]\right]=1
$$

unless $\tau \in K_{1} R^{*}$; and $K_{1} R^{*} \cap T=R^{*}$. Finally, note that every element of $R^{*}$ is even. Using this information, we find that

$$
\begin{aligned}
\prod_{\sigma \in S}\left[u_{2 n}, u_{(2 n-1) \sigma}, \cdots, u_{1 \sigma}\right]^{\epsilon(\sigma)} \\
=\prod_{\rho^{*} \in R^{*}}\left[\left[u_{2 n}, u_{(2 n-1) \rho^{*}}\right], \cdots,\left[u_{2 \rho^{*}}, u_{1 \rho^{*}}\right]\right] \\
=\prod_{\rho \in R}\left[v_{n}, v_{(n-1) \rho}, \cdots, v_{1_{\rho}}\right] \\
=\prod_{r=1}^{n-1}\left[v_{n}, v_{r}, v_{1}, \cdots, v_{r-1}, v_{r+1}, \cdots, v_{n-1}\right]^{(n-2) !},
\end{aligned}
$$

where the last equality holds because $W_{n}$ is metabelian. By $[3,36.32]$, this value of $w_{2 n}$ is not trivial. This proves that if $c$ is even, $w_{c}$ is not a law in $G_{c}$.

A similar argument shows that if $c=2 n-1$ and $u_{i}$ is substituted for $x_{i}$, the value of $w_{2 n-1}$ is

$$
\left.\prod_{r=1}^{n-1}\left[u_{2 n-1}, v_{r}, v_{1}, \cdots, v_{r-1}, v_{r+1}, \cdots, v_{n-1}\right]\right]^{(n-2) !}
$$

which does not belong to the $2 n$th term of the lower central series of $H_{n}$. Thus also when $c$ is odd, $w_{c}$ is not a law in $G_{c}$, and this completes the outline of the proof.

REMARK (added in proof July 9, 1968). An alternative and independent proof of this result has been obtained by Professor F. Levin and submitted to J. Austral. Math. Soc.

\section{REFERENCES}

1. Graham Higman, Representations of general linear groups and varieties of $p$ groups, Proc. Internat. Conf. Theory of Groups, Australian National University, Canberra, August 1965, Gordon and Breach, New York, 1967, pp. 167-173.

2. - A remark on finitely generated nilpotent groups, Proc. Amer. Math. Soc. 6 (1955), 284-285.

3. Hanna Neumann, Varieties of groups, Springer, Berlin, 1967.

4. Martin Ward, Basic commutators, submitted to Philos. Trans. Roy. Soc. London Ser. A.

Australian National University, Canberra 